The finding that below $85 \%$ adherence, readmission is significantly more likely suggests that there may be a role for carefully and progressively monitoring depot adherence in community services. Reduction in relapses from enhanced adherence will have clinical, social and economic benefits.

\section{The metabolic syndrome in patients with a prolonged psychotic illness within a community setting: age and gender issues}

\author{
T Lambert ${ }^{1,2,3}$, C Pantelis ${ }^{4}$, N Freeman' \\ ${ }^{1}$ Office for Psychiatric Evaluation and Educational NewMedia (OPEN); ${ }^{20 R Y G E N ; ~}$ \\ ${ }^{3}$ The University of Melbourne ${ }^{4}$ Melbourne Neuropsychiatry Centre, \\ The University of Melbourne, Melbourne, Australia
}

Background: To measure the prevalence of the metabolic syndrome among patients with a prolonged psychotic illness being treated within a community setting and to examine gender- and age-stratified trends.

Methods: The study sample consisted of patients receiving treatment in the midwest or northwest mental health regions of Melbourne, Australia, between February 2003 and February 2004. Of the 206 patients aged 18 years and over who were approached, 106 consented to participate, yielding a response rate of $52.7 \%$. Participants were assessed for the presence of metabolic syndrome using the Adult Treatment Panel Guidelines-III).

Results: Prevalence of the metabolic syndrome among patients within this population was $39.4 \%$ (an alternate method of determining the denominator, indicates a rate of $\sim 50 \%$ ). Patients with the metabolic syndrome had a higher body mass index (31.73 vs. 28.82, $P<0.01)$ and increased triglyceride levels (3.49 vs. $1.70, P<0.01)$ compared with patients without this syndrome. Men with the metabolic syndrome had lower mean high-density lipoprotein cholesterol levels $(0.95$ vs. $1.24, P<0.01)$ compared with men without this syndrome. In general, there were distinct gender patterns of abnormality in the metabolic components, and age stratification shows increased relative risks in the young as contrasted with the older patients.

Conclusions: The prevalence of the metabolic syndrome is high among people with a prolonged psychotic illness. Health professionals treating people within this population need to be monitoring their patients' physical health as well as looking after their mental well-being.

\section{Stability of antipsychotic prescribing: description and relationship to readmission}

\author{
T Lambert ${ }^{1,2}$, B Singh ${ }^{2}$ \\ 'OPEN/ORYGEN; and ${ }^{2}$ The University of Melbourne, Melbourne, Australia
}

Background: As showed by the CATIE study, antipsychotic prescribing/switching stability appears to be less than robust. Little is known of longitudinal stability in other treatment cultures. This paper presents Australian data to outline trends in routine clinical practice of CCT-treated patients.

Methods: A cohort abstracted from our large database is described. Prescribing stability and readmission were examined in patients with schizophrenia treated with antipsychotic monotherapy at $\mathrm{T} 1$ and $\mathrm{T} 2$ (18 months panel data). Of the 817 patients, 302 were on monotherapy at both times.

Results: A matrix of prescribing/switching stability indicating the rate of persistence on one medicine and, if switched, to what antipsychotic will be presented. Eighteen-month monotherapy persistence rates were as follows: clozapine $81.3 \%$, olanzapine $71.0 \%$, FGA depots $65.3 \%$, risperidone $51.4 \%$ and FGA orals $34.0 \%$. Numerically, the largest switching traffic occurred from depot to olanzapine and vice versa. Readmission, only $13.4 \%$ were readmitted. Being on a depot at T1 was 2.33 times more likely than being on an oral to result in admission in the study period $(P=0.004)$. If clozapine is excluded, there is no difference between depots and SGAs (RR 1.62, $P>0.05$ ). Overall readmission was 2.15 times more likely to occur in those in whom antipsychotic switching occurs (direction of causality undetermined).

Conclusions: Stability is somewhat higher in Australia than reported for the United States. However, the general comparative trends in terms of the various antipsychotics are supported. Clozapine and olanzapine appear to have particularly stable use.

\section{Psychostimulant withdrawal: natural history and options for intervention}

\section{N Lee, A Harney, L Johns, A Pennay, P Kenny}

Turning Point Drug and Alcohol Centre, Melbourne, Australia

Background: Psychostimulant withdrawal is still not well understood. Much of the limited literature has been in the cocaine area and very little with methamphetamine. In particular, the natural history of withdrawal from psychostimulants is not well documented 